\title{
Red-shifted $\mathrm{H}_{2} \mathrm{O}$ emission in NGC 3079: More evidence for a pc-scale circumnuclear torus? ${ }^{\star}$
}

\author{
Y. Hagiwara ${ }^{1,2}$, C. Henkel ${ }^{1}$, W. A. Sherwood ${ }^{1}$, and W. A. Baan ${ }^{2}$ \\ 1 Max-Planck-Institut für Radioastronomie, Auf dem Hügel 69, 53121 Bonn, Germany \\ ${ }^{2}$ Westerbork Observatory, PO Box 2, 7990 AA Dwingeloo, The Netherlands \\ Received 23 January 2002 / Accepted 2 April 2002
}

\begin{abstract}
Using the Effelsberg 100-m telescope, sensitive measurements of the $\mathrm{H}_{2} \mathrm{O}$ megamaser in NGC 3079 are presented. During 2000-2001, "high velocity" features are seen that are red-shifted up to $225 \mathrm{~km} \mathrm{~s}^{-1}$ with respect to the systemic velocity of the galaxy $\left(V_{\mathrm{LSR}} \sim 1120 \mathrm{~km} \mathrm{~s}^{-1}\right)$. Symmetrically bracketing the systemic velocity, the $\mathrm{H}_{2} \mathrm{O}$ emission covers a velocity range of $\sim 450 \mathrm{~km} \mathrm{~s}^{-1}$ with only one potential narrow gap $\left(\sim 20 \mathrm{~km} \mathrm{~s}^{-1}\right)$ near the systemic velocity itself. Velocity drifts of individual components are not convincingly detected. It is shown that the presence of red-shifted emission and the absence of detectable velocity drifts are not inconsistent with the existence of a rotating circumnuclear maser disk at the very center of the galaxy. Significant differences in the overall line profile compared to NGC 4258 and a complex morphology of the radio continuum leave, however, space for scepticism.
\end{abstract}

Key words. galaxies: active - galaxies: individual: NGC 3079 - galaxies: ISM - galaxies: nuclei radio lines: galaxies

\section{Introduction}

Recent single-dish and VLBI observations of luminous $\mathrm{H}_{2} \mathrm{O}$ megamasers have been motivated by the discovery of an edge-on Keplerian sub-pc scale maser disk enshrouding a compact supermassive object at the nucleus of the LINER galaxy NGC 4258 (e.g. Nakai et al. 1993, 1995; Haschick et al. 1994; Greenhill et al. 1995; Miyoshi et al. 1995; Herrnstein et al. 1999). Circumnuclear disk structures traced by $\mathrm{H}_{2} \mathrm{O}$ are also seen towards NGC 1068, NGC 4945, and the Circinus galaxy (e.g. Gallimore et al. 2001; Greenhill et al. 1997, 2000). $\mathrm{H}_{2} \mathrm{O}$ megamasers therefore provide a unique probe to study the kinematics and the dynamical structure of the innermost regions of active galactic nuclei.

For the LINER or Seyfert 2 galaxy NGC 3079 (see Sawada-Satoh et al. 2000) that contains one of the most luminous $\mathrm{H}_{2} \mathrm{O}$ megamasers known to date (e.g. Henkel et al. 1984; Haschick \& Baan 1985; Haschick et al. 1990), arguments in favor of a circumnuclear disk have been less compelling. While the known maser components arise in the inner few parsecs of the galaxy, they do not align at right angles to the radio jet(s), almost all the detected emission is blue-shifted relative to the systemic velocity, and any velocity drift of individual $\mathrm{H}_{2} \mathrm{O}$ features must be small

Send offprint requests to: Y. Hagiwara, Dwingeloo

* Based on observations with the 100-m telescope of the MPIfR (Max-Planck-Institut für Radioastronomie) at Effelsberg.
( $\$ 4.0 \mathrm{~km} \mathrm{~s}^{-1} \mathrm{yr}^{-1}$; Nakai et al. 1995; Baan \& Haschick 1996; Trotter et al. 1998; Satoh et al. 1999; Sawada-Satoh et al. 2000).

In this letter we therefore report single-dish observations of the $22 \mathrm{GHz}$ water vapor line from NGC 3079, (1) to look for the missing red-shifted velocity components and (2) to search for velocity drifts in the near systemic features that would indicate centripetal acceleration in a rotating circumnuclear disk.

\section{Observations}

Observations of the $J_{K_{\mathrm{a}} K_{\mathrm{c}}}=6_{16}-5_{23} \quad \mathrm{H}_{2} \mathrm{O}$ maser line (rest frequency: $22.23508 \mathrm{GHz}$ ) were made with the MPIfR 100-m radio telescope at Effelsberg between February 1994 and December 2001. Until 1998, a single channel $K$-band maser receiver was employed in a position switching mode with a system temperature of $T_{\text {sys }} \sim 225 \mathrm{~K}$ on a main beam brightness temperature $\left(T_{\mathrm{mb}}\right)$ scale. An autocorrelator provided bandwidths of 50,25 or $12.5 \mathrm{MHz}$ with 1024 spectral channels, yielding channel spacings of 0.66 , 0.33 or $0.16 \mathrm{~km} \mathrm{~s}^{-1}$. Since 2000 , we used a dual channel $K$-band HEMT receiver in a dual beam switching mode with a beam throw of $2^{\prime}$ and a switching frequency of $1 \mathrm{~Hz}$. After averaging the two orthogonally polarized signals, $T_{\text {sys }} \sim 180 \mathrm{~K}$. Four autocorrelator backends were employed for each receiver channel. Each spectrum had a bandwidth of $40 \mathrm{MHz}$ and 512 channels, yielding a channel spacing of $1.05 \mathrm{~km} \mathrm{~s}^{-1}$. 
Table 1. Effelsberg $100-\mathrm{m}$ observations $\log ^{\mathrm{a})}$.

\begin{tabular}{llr|lll}
\hline \hline Epoch & & & Epoch & & \\
\hline 1994 & Feb. & 9 & 2000 & Mar. & 18 \\
1995 & Sep. & $16-17$ & 2000 & Oct. & 14 \\
1996 & Sep. & $21-23$ & 2000 & Dec. & 21 \\
1997 & Apr. & $5-6$ & 2000 & Mar. & 13 \\
1997 & Nov. & 5 & 2001 & Apr. & 22 \\
1998 & Feb. & 1 & 2001 & May & 9 \\
1998 & May & 8 & 2001 & Dec. & 5 \\
1998 & Jun. & 27 & & & \\
1998 & Aug. & 1 & & & \\
\hline \multicolumn{7}{c}{}
\end{tabular}

a) Several tunings and bandwidths were employed during most epochs. As a consequence, noise levels and channel spacings are not uniform across the observed velocity ranges. $1 \sigma$ noise levels vary from $200 \mathrm{mJy}$ (channel spacing $1.32 \mathrm{~km} \mathrm{~s}^{-1}$ ) in September 1996 to $7 \mathrm{mJy}$ (channel spacing $1.05 \mathrm{~km} \mathrm{~s}^{-1}$ ) in December 2001. Velocity resolution $\sim 1.25 \times$ channel spacing. For direct access to 64 flux calibrated spectra, contact chenkel@mpifr-bonn.mpg.de.

Amplitude calibration was based on measurements of the $22 \mathrm{GHz}$ continuum flux of $3 \mathrm{C} 286$ (see Baars et al. 1977; Ott et al. 1994). Pointing measurements toward nearby sources (in most cases DA 251) were made once per hour. The resultant pointing accuracy was $<8^{\prime \prime}$, that should be compared with the full width to half power beam size of $40^{\prime \prime}$. Calibration uncertainties are estimated to be $\pm 15 \%$.

\section{Results}

$\mathrm{H}_{2} \mathrm{O}$ spectra were taken during 16 epochs (Table 1 ; no changes in the maser profiles were seen within any of these observing periods). Several $\mathrm{H}_{2} \mathrm{O}$ features could be traced over the entire monitoring period. The most prominent component, detected at $\sim 956 \mathrm{~km} \mathrm{~s}^{-1}$, showed a peak flux density of $2.5-4.5 \mathrm{Jy}$. While the peak velocity is observed to drift from about 955.0 to $956.8 \mathrm{~km} \mathrm{~s}^{-1}$ (estimated error of individual measurements: $0.3 \mathrm{~km} \mathrm{~s}^{-1}$ ), this drift is not systematic and appears to be caused by a multitude of individual, variable subcomponents. During times with lower peak velocity, the profile tends to show a "shoulder" at the high velocity wing of the line (e.g., on Nov. 5, 1997 and Feb. 1, 1998) and vice versa (Dec. 21, 2000, and Apr. 22, 2001). Notable were two flares of more red-shifted components that exceeded $2 \mathrm{Jy}$ : The $979 \mathrm{~km} \mathrm{~s}^{-1}$ component with 0.7 Jy during Feb. 1994 to Apr. 1997 (see Nakai et al. 1995 for earlier spectra) reached 0.9 Jy in Nov. 1997, 1.5 Jy in Feb. 1998, and 2.2 Jy in May 1998 (Fig. 1a); it then decreased to $1.8 \mathrm{Jy}$ in June 1998, 1.25 Jy in Aug. 1998, and 0.35 Jy since Oct. 2000. The $1017 \mathrm{~km} \mathrm{~s}^{-1}$ velocity component, observed between Sep. 1996 and Nov. 1997 at 0.1-0.5 Jy, reached a peak flux density of $2.5 \mathrm{Jy}$ in Mar. 2000 (Fig. 1b); flux densities decreased to $1.0 \mathrm{Jy}$ in Oct. 2000 and to $0.1 \mathrm{Jy}$

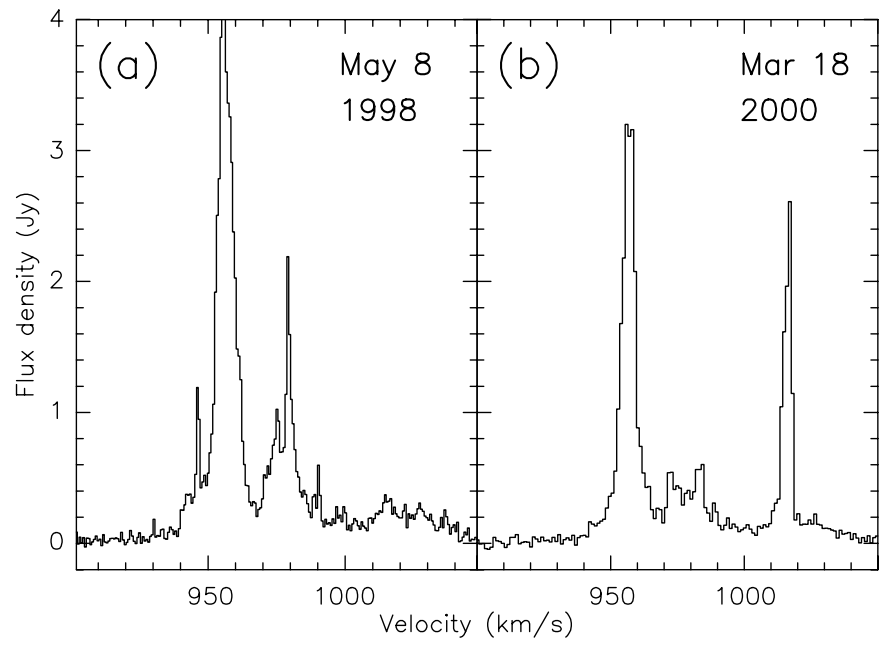

Fig. 1. The a) 979 and b) $1017 \mathrm{~km} \mathrm{~s}^{-1}$ features flaring beyond the 2 Jy level (channel spacings: 0.66 and $1.05 \mathrm{~km} \mathrm{~s}^{-1}$ ). Velocity scale throughout the paper: Local Standard of Rest (LSR), following the radio astronomical definition of velocity (see e.g. Trotter et al. 1998). $V_{\mathrm{LSR}}=V_{\mathrm{HEL}}+3.23 \mathrm{~km} \mathrm{~s}^{-1}$.

in May 2001. Emission near the systemic velocity of the galaxy $\left(1080 \mathrm{~km} \mathrm{~s}^{-1}<V_{\mathrm{LSR}}<1160 \mathrm{~km} \mathrm{~s}^{-1}\right)$ remained faint and not a single narrow velocity component could be traced during several consecutive observing epochs.

Figure 2 shows a spectrum of near systemic $\left(V_{\text {sys }} \sim\right.$ $\left.1120 \mathrm{~km} \mathrm{~s}^{-1}\right)$ and red-shifted $\left(V>V_{\text {sys }}\right)$ features that were detected since March 2000. More than 10 distinct narrow components appear at velocities in excess of $1100 \mathrm{~km} \mathrm{~s}^{-1}$ (Table 2). We conclude that the $\mathrm{H}_{2} \mathrm{O}$ maser emission extends over a radial velocity range of $\sim 450 \mathrm{~km} \mathrm{~s}^{-1}$. The integrated luminosity of the previously known blue-shifted components remains approximately constant. This may also hold for the systemic and red-shifted features shown in Fig. 2. A detection of these prior to 2000 would have been difficult in view of technical improvements that occurred at Effelsberg in 1999. Sensitivity also limits the studies by Nakai et al. (1995; we do not confirm the weak 764 and $791 \mathrm{~km} \mathrm{~s}^{-1}$ components) and Baan \& Haschick (1996). However, the 1123 and $1190 \mathrm{~km} \mathrm{~s}^{-1}$ components observed by Trotter et al. (1998) in Jan. 1995, the flaring $1192 \mathrm{~km} \mathrm{~s}^{-1}$ feature ( 0.4 Jy) detected by Nakai et al. (1995) in Apr.-May 1995, and a $1201 \mathrm{~km} \mathrm{~s}^{-1}$ feature detected by us in Sep. 1995 (Fig. 3) indicate the presence of isolated red-shifted maser features as early as half a dozen years ago.

\section{Discussion}

In NGC 4258, Nakai et al. (1993) detected red- and blue-shifted $\mathrm{H}_{2} \mathrm{O}$ "satellites" symmetrically displaced by approximately $\pm 900 \mathrm{~km} \mathrm{~s}^{-1}$ from the systemic velocity. This suggested the presence of a circumnuclear disk that was later confirmed (see Sect.1). Is the discovery of a multitude of near systemic and red-shifted features in NGC 3079 also hinting at a circumnuclear disk? Emission is seen at least between 928 and $1352 \mathrm{~km} \mathrm{~s}^{-1}$ (Fig. 3). 


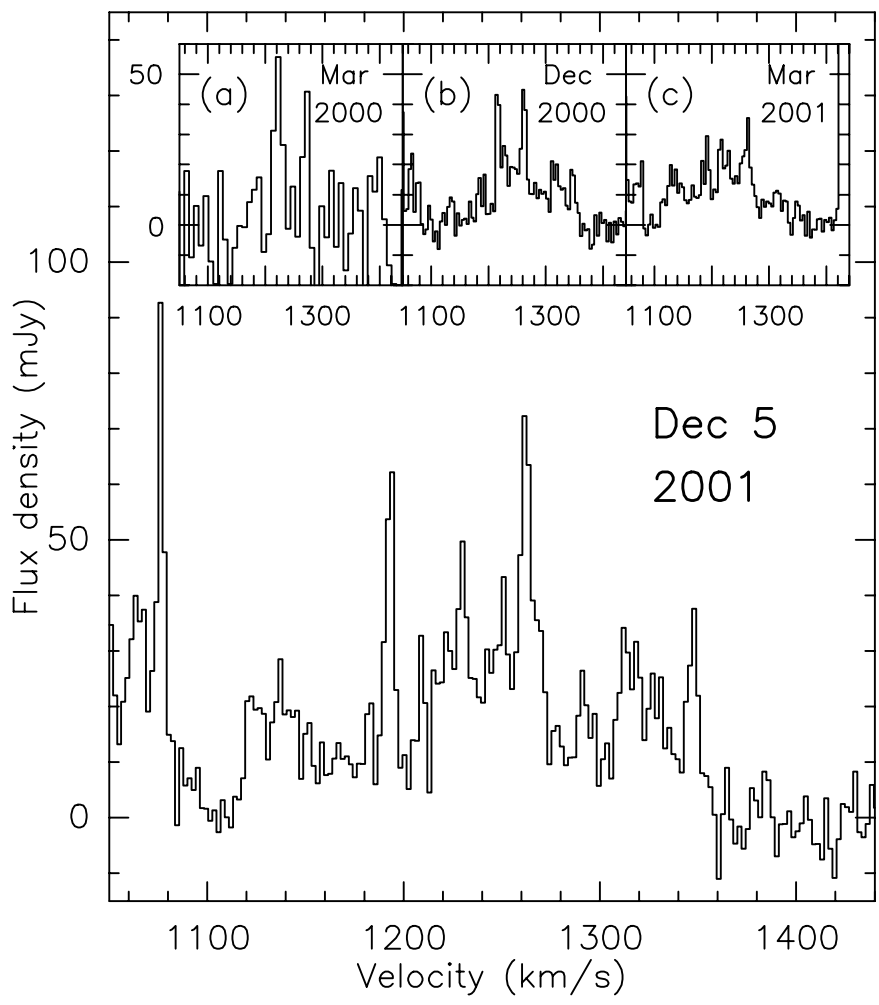

Fig. 2. Near systemic and red-shifted $\mathrm{H}_{2} \mathrm{O}$ maser features toward NGC 3079. Channel spacing: $2.1 \mathrm{~km} \mathrm{~s}^{-1}$ (main spectrum), $4.2 \mathrm{~km} \mathrm{~s}^{-1}$ (inserts b) and c)), and $8.4 \mathrm{~km} \mathrm{~s}^{-1}$ (insert a)). The inserted spectra (insert c) includes a few bad channels at the edge of the band) show an initially dominant fading $1222 \mathrm{kms}^{-1}$ component, while the flux of the $1192 \mathrm{~km} \mathrm{~s}^{-1}$ component increases. The brightest components have an isotropic luminosity of $\sim 0.5 L_{\odot}$. Since the zero level is determined by a linear baseline derived from velocities $V<920$ and $>1360 \mathrm{~km} \mathrm{~s}^{-1}$, the absence of $\mathrm{H}_{2} \mathrm{O}$ emission near $1100 \mathrm{~km} \mathrm{~s}^{-1}$ is not entirely certain.

Table 2. Velocities of distinct near systemic or red-shifted features observed on December 5, 2001 (see Fig. 2).

\begin{tabular}{cc}
\hline \hline$V\left(\mathrm{~km} \mathrm{~s}^{-1}\right)$ & $V\left(\mathrm{~km} \mathrm{~s}^{-1}\right)$ \\
\hline $1062.7 \pm 0.4$ & $1209.3 \pm 0.3$ \\
$1066.8 \pm 1.4$ & $1229.8 \pm 0.4$ \\
$1076.5 \pm 0.2$ & $1251.1 \pm 0.1$ \\
$1123.0 \pm 0.9$ & $1262.1 \pm 0.2$ \\
$1137.7 \pm 1.4$ & $1291.5 \pm 0.9$ \\
$1182.6 \pm 0.4$ & $1314.4 \pm 1.0$ \\
$1192.9 \pm 0.5$ & $1347.6 \pm 0.4$ \\
\hline
\end{tabular}

Assuming the presence of a symmetric edge-on circumnuclear disk, the systemic velocity of the nuclear region then is $\sim 1140 \mathrm{~km} \mathrm{~s}^{-1}$. Including the $896 \mathrm{~km} \mathrm{~s}^{-1}$ feature apparent in the early spectra of Henkel et al. (1984) and Haschick \& Baan (1985) and tentatively also seen in Dec. 2000 and Dec. 2001, $V_{\text {sys }} \sim 1124 \mathrm{~km} \mathrm{~s}^{-1}$. Is this consistent with the systemic velocity of the galaxy?
From the nuclear molecular gas traced by the CO $J=$ 1-0 line, Irwin \& Sofue (1992) deduced a systemic velocity of $V_{\text {sys }}=1050 \pm 10 \mathrm{~km} \mathrm{~s}^{-1}$. From an H i line profile, Irwin \& Seaquist (1991) obtained a midpoint at the $20 \%$ peak level of $1123 \pm 10 \mathrm{~km} \mathrm{~s}^{-1}$ and $1126 \mathrm{~km} \mathrm{~s}^{-1}$ from a fit to the moment map. Three dimensional modeling of their H I data cube yields $V_{\text {sys }}=1116 \mathrm{~km} \mathrm{~s}^{-1}$. The rough agreement of $\mathrm{CO}, \mathrm{HI}$, and $\mathrm{H}_{2} \mathrm{O}$ velocity centroids implies a nuclear systemic velocity of $\sim 1120 \mathrm{~km} \mathrm{~s}^{-1}$ and suggests that we have seen, for the first time, all the stronger $22 \mathrm{GHz}_{2} \mathrm{O}$ maser components in NGC 3079. A nuclear systemic velocity of $1230 \mathrm{~km} \mathrm{~s}^{-1}$ as suggested by Satoh et al. (1999) is not supported by our $\mathrm{H}_{2} \mathrm{O}$ data.

Nevertheless, the maser emission is far from perfectly symmetric: (1) All bright components (>0.5 Jy) are blueshifted with respect to the systemic velocity which is inconsistent with the situation in NGC 4258 and NGC 1068 as well as with the spiral shock model proposed by Maoz \& McKee (1998). (2) The systemic velocity obtained from model fits (Irwin \& Seaquist 1991) lies at the edge of that narrow velocity interval $\left(\Delta V \sim 20 \mathrm{~km} \mathrm{~s}^{-1}\right.$, see Fig. 2) that appears to be devoid of $\mathrm{H}_{2} \mathrm{O}$ emission (the only such interval over the entire $\mathrm{H}_{2} \mathrm{O}$ velocity range observed by us). Interpreted in terms of the paradigm established for NGC 4258, this may hint at a lack of nuclear $22 \mathrm{GHz}$ radio continuum emission at the very center of the putative masering torus. (3) In a well ordered edge-on circumnuclear disk, small line-of-sight velocity gradients that stimulate maser emission should only be present near the tangential points and near the front and back side of the disk. A continuous coverage of the velocity range as observed in NGC 3079 is not expected. (4) None of the four systemic velocities quoted above provides a particularly high number of red- and blue-shifted components that match each other with respect to velocity $\left(\left|V_{\text {red }}-V_{\text {sys }}\right| \sim\left|V_{\text {blue }}-V_{\text {sys }}\right|\right)$. Note that the following discussion, does not sensitively depend on the exact choice of $V_{\text {sys }}$ as long as it is in the range $1100-1150 \mathrm{~km} \mathrm{~s}^{-1}$.

Most blue-shifted $22 \mathrm{GHz} \mathrm{H}_{2} \mathrm{O}$ velocity components of NGC 3079 are already known to show no significant velocity drift (e.g. Fig. 7 of Nakai et al. 1995; Fig. 6 of Baan $\&$ Haschick 1996). The slow drift of $\sim 0.4 \mathrm{~km} \mathrm{~s}^{-1} \mathrm{yr}^{-1}$ suggested by Baan \& Haschick (1996) for the 945, 951, and $1015 \mathrm{~km} \mathrm{~s}^{-1}$ components ( $V$ refers to epoch 1994.0) is not expected if a circumnuclear torus is present (e.g. Miyoshi et al. 1995). Our data for the $945 \mathrm{~km} \mathrm{~s}^{-1}$ component (945.7 and $946.2 \mathrm{~km} \mathrm{~s}^{-1}$ in Feb. 1994 and 1998, and 946.6 and $946.5 \mathrm{~km} \mathrm{~s}^{-1}$ in May and June 1998; errors derived from Gaussian fits are $\sim 0.3 \mathrm{kms}$ ) are not contradicting Baan \& Haschick (1996), although a group of features with varying amplitudes could also simulate the drift. The $1017 \mathrm{~km} \mathrm{~s}^{-1}$ component (Fig. 1b) fluctuates between 1014 and $1018 \mathrm{~km} \mathrm{~s}^{-1}$ and does not support a regular drift, although it is likely related to the $1015 \mathrm{~km} \mathrm{~s}^{-1}$ component of Baan \& Haschick (1996). The $951 \mathrm{~km} \mathrm{~s}^{-1}$ component was not seen by us. Satoh et al. (1999) and SawadaSatoh et al. (2000) report a drift of $3.7 \pm 0.6 \mathrm{~km} \mathrm{~s}^{-1} \mathrm{yr}^{-1}$ for a maser feature near $1190 \mathrm{~km} \mathrm{~s}^{-1}$. We find a group 


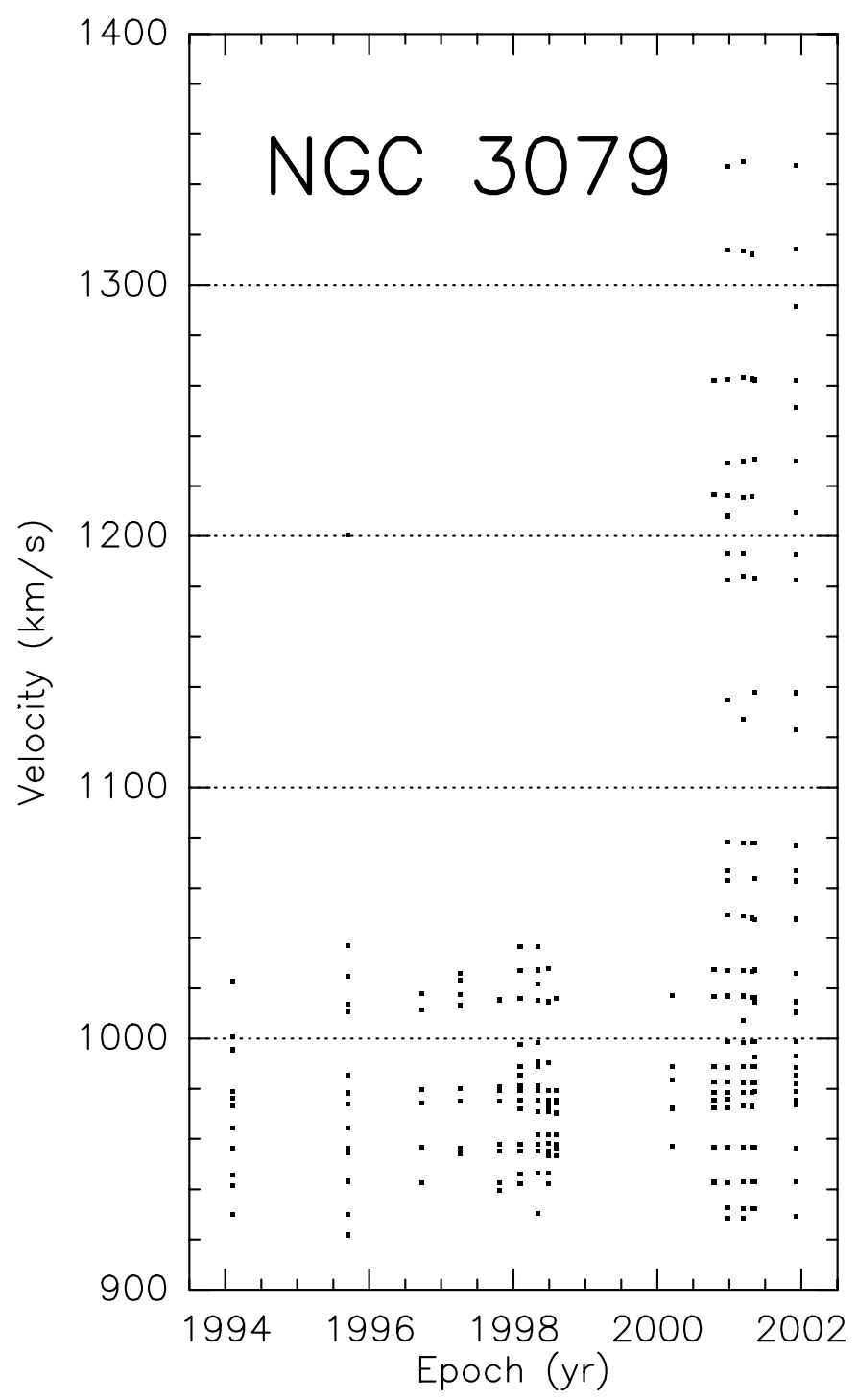

Fig. 3. Individual velocity components of the $22 \mathrm{GHz} \mathrm{H}_{2} \mathrm{O}$ spectrum obtained with an accuracy $\lesssim 1 \mathrm{~km} \mathrm{~s}^{-1}$ for the blueshifted components and $\sim 1 \mathrm{~km} \mathrm{~s}^{-1}$ for the redshifted ones.

of features (total width: $V_{\text {tot }} \sim 5-6 \mathrm{~km} \mathrm{~s}^{-1}$ ) centered at $1192.9 \mathrm{kms}^{-1}$, both on Mar. 3 and Dec. 5, 2001. Thus the drift appears to be well below our estimated detection level of $1.5 \mathrm{~km} \mathrm{~s}^{-1} \mathrm{yr}^{-1}$ for a time interval of 9 months. In view of the long lifetime inferred for some of the blueshifted maser lines (Sect. 3 and Fig. 3), the $1192 \mathrm{~km} \mathrm{~s}^{-1}$ feature reported by Nakai et al. (1995) is likely part of this component, reducing the velocity drift to well below $1.0 \mathrm{~km} \mathrm{~s}^{-1} \mathrm{yr}^{-1}$.

The overall lineshape of the $\mathrm{H}_{2} \mathrm{O}$ profile is quite distinct from that of NGC 4258 and the morphology of the radio continuum ( $\mathrm{T}$. Krichbaum, priv. comm.) is more complex. In spite of the presence of a roughly linear ridge of blue-shifted $\mathrm{H}_{2} \mathrm{O}$ masers, the existence of an associated nuclear disk is thus not certain (see e.g. Trotter et al. 1998 for alternative scenarios). Sawada-Satoh et al. (2000) proposed a thick maser disk with continuum source "B" at the nucleus and the $1190 \mathrm{~km} \mathrm{~s}^{-1}$ feature on its near side (their Fig.6). Since we see no velocity drift near $1190 \mathrm{kms}^{-1}$ and since this is not the systemic velocity, however, there is no need to put this component at the near side of the putative disk. We therefore tend to favor the disk scenario outlined by Trotter et al. (1998) with the nucleus of the galaxy being located between continuum components A and B (their Fig.7). In this latter scenario, expected velocity drifts would be consistent with our upper limits. The nucleus would contain a few $10^{6} M_{\odot}$ within $R \sim 10$ mas $(\sim 0.7 \mathrm{pc}$; assumed rotation velocity $V_{\text {rot }} \sim 100-225 \mathrm{~km} \mathrm{~s}^{-1}$ ), the centripetal acceleration and proper motion of the near-systemic components would be difficult to detect $\left(<0.1 \mathrm{~km} \mathrm{~s}^{-1} \mathrm{yr}^{-1}\right.$ and $\left.\sim 1 \mu \mathrm{as} \mathrm{yr}^{-1}\right)$, and most of the red-shifted masers would be located 10-15 mas south of the blue-shifted ones (some masers may be associated with the jet (e.g. Trotter et al. 1998)). Sensitive VLBI data are needed to examine this picture and to obtain definite information on the relative location of blueand red-shifted maser features and continuum sources in the very center of NGC 3079.

Acknowledgements. We wish to thank M. Inoue and L. J. Greenhill for critically reading the manuscript.

\section{References}

Baan, W. A., \& Haschick, A. D. 1996, ApJ, 473, 269

Baars, J. W. M., Genzel, R., Pauliny-Toth, I. I. K., \& Witzel, A. 1977, A\&A, 61, 99

Gallimore, J. F., Henkel, C., Baum, S. A., et al. 2001, ApJ, 556,694

Greenhill, L. J., Henkel, C., Becker, R., Wilson, T. L., \& Wouterloot, J. G. A. 1995, A\&A, 304, 21

Greenhill, L. J., Moran, J. M., \& Herrnstein, J. R. 1997, ApJ, 481, L23

Greenhill, L. J. 2000, Proc. of the 5th European VLBI Network Symp., ed. J. E. Conway, A. G. Polatidis, R. S. Booth, \& Y. M. Pihlström (Onsala), 101

Haschick, A. D., \& Baan, W. A. 1985, Nature, 314, 144

Haschick, A. D., Baan, W. A., Schneps, M. H., et al. 1990, ApJ, 356, 149

Haschick, A. D., Baan, W. A., \& Peng, E. W. 1994, ApJ, 437, L35

Henkel, C., Güsten, R., Downes, D., et al. 1984, A\&A, 141, L1

Herrnstein, J. R., Moran, J. M., Greenhill, L. J., et al. 1999, Nature, 400, 539

Irwin, J. A., \& Seaquist, E. R. 1991, ApJ, 371, 111

Irwin, J. A., \& Sofue, Y. 1992, ApJ, 396, L75

Maoz, E., \& McKee, C. F. 1998, ApJ, 494, 218

Miyoshi, M., Moran, J. M., Herrnstein, J., et al. 1995, Nature, 373,127

Nakai, N., Inoue, M., \& Miyoshi, M. 1993, Nature, 361, 45

Nakai, N., Inoue, M., Miyazawa, K., Miyoshi, M., \& Hall, P. 1995, PASJ, 47, 771

Ott, M., Witzel, A., Quirrenbach, A., et al. 1994, A\&A, 284, 331

Satoh, S., Inoue, M., Nakai, N., et al. 1999, Adv. Sp. Res., 23, 1025

Sawada-Satoh, S., Inoue, M., Shibata, K. M., et al. 2000, PASJ, 52,421

Trotter, A. S., Greenhill, L. J., Moran, J. M., et al. 1998, ApJ, 495,740 